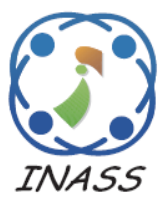

\title{
An Energy Efficient Clustering Using K-Means and AODV Routing Protocol in Ad-hoc Networks
}

\author{
Bandani Anil Kumar ${ }^{1 *}$ \\ Makam Venkata Subramanyam² \\ Kodati Satya Prasad ${ }^{1}$ \\ ${ }^{1}$ Jawaharlal Nehru Technological University, Kakinada India \\ ${ }^{2}$ Santhiram Engineering College, Nandyal, India \\ * Corresponding author's Email: anilbandani@gmail.com
}

\begin{abstract}
Mobile Ad-hoc Network (MANET) is a collection of wireless mobile hosts that create a temporary network without the help of centralized administration or particular support services. Energy consumption is the most critical issue in the MANETs, since the superior part of the mobile hosts work on inhibited battery resources. Reduction in the energy consumption results in the increase in the lifetime and throughput of the network. The performance of existing strategies is less in terms of energy conservation issues. To overcome these limitations, this paper uses an energy consumption method incorporate with a proactive MANET routing protocol. The routing protocol depends on the energy level and the movement of the nodes. Estimation of energy cost is performed based on the calculation of the energy consumption level of the node, using K-Means clustering along with AODV (Adhoc On Demand Distance Vector) optimized with Ant Colony Optimization (ACO). The performance of the proposed "K-Means-AODV-ACO" model is compared with the existing Energy Efficient Hybrid Routing Protocol (EE-HRP) and Mobile Agents based Reliable and energy efficient routing protocol (MAREERP) MANETs. From the comparison result, it is clearly seen that the proposed model accomplished better execution in terms of number of Packet delivery ratio (PDR), end-to-end delay, delay, drop and energy consumption.
\end{abstract}

Keywords: Ant colony optimization, Ad-hoc on demand distance vector, Centralized administration, End-to-end delay, Mobile ad-hoc network, Packet delivery ratio, Secure data transmission.

\section{Introduction}

Mobile ad-hoc network (MANET) is designed with mobile wireless nodes, which communicate over the wireless links of the distributed fashion in the absence of centralized access point or fixed physical infrastructure [1]. Ant Colony Optimization (ACO) technique chooses the multiple routes of MANET based on the maximum path preference [2]. Link and node instability of MANET avoided by developing the multipath multicast routing with the benefit of reliable neighbour selection mechanism [3]. The Residual-Energy based Reliable Multicast Routing (RERMR) protocol maintains the reliable multicast routing that improves the connectivity and stability of the network and this RERMR protocol mainly depends on the threshold value [4]. An energy efficient routing algorithm Secure Three Fish
Distributed Meaning (STFDR) creates the route from source to destination that provides the collision awareness and security [5]. The self-adaptive genetic algorithm developed for routing between the nodes under the dynamic topology environment [6].

In power heterogeneous MANETs, the free virtual clustering based RP presented for minimizing the obstruction caused by high power nodes and Authenticated Anonymous Secure Routing (AASR) developed for defending against potential active anonymous attacks [7-8]. In mobility state adaptive routing, the routing is achieved by using either ETX or the MF metric, which is identified based on a detected mobility state. The PDR model doesn't show better results in both static and mobility scenarios while reducing the number of dropped packet [9]. The information about the local link connectivity is identified by an adaptive HELLO messaging scheme to observe the link status among 
the nodes as well as the dynamic on demand routing protocol is utilized for minimizing the energy consumption. Then this adaptive HELLO messaging method reduced the unwanted hello messages. The Hello interval can't be enlarged without reduced detectability of a broken link and affect overhead and energy. [10 - 11]. An energy efficient route over the MANET is made by utilizing a vague set estimation strategy in an energy effective RP. The interval based membership function is used in the vague set measurement technique. The network lifetime based on the different parameters as vague set and deciding some fitness threshold statistic are not mentioned [12].

In the Temporally Ordered Routing AlgorithmRouting Protocol (TORA-RP), the Binary Particle Swarm Optimization (BPSO) algorithm includes the element of the energy awareness. This BPSO-TORA considers the routes length and energy level while establishing the route. The total delivered data, network lifetime and system lifetime are low [13]. AODV based clustering and routing is presented in the MANET and this protocol is used for integrated routing and message delivery complete the clustered networks. The algorithm didn't provide more speed and transmission rate in the simulation [14]. An Anonymous Location based Efficient RP (ALERT) is declared for proposing high anonymity security at low cost. If the delay increase, it affects the overall presentation of the network. The reinforcing ALERT in an attempt, active attackers are not up to level [15]. The Ad-hoc on demand multipath distance vector used a fitness function (FFAOMDV) to generate the optimum path from source to destination. The fitness function considers only two parameters such as distance and energy. The fitness value with bandwidth as another fitness value is not mentioned [16]. To overcome the above mentioned problems the k-means clustering with ACO optimized AODV routing for selecting the optimal path to transfer the data over the network is introduced. Hence, "K-Means-AODV" methodology provides better results in delay (6\%), DelRatio (7\%), energy consumption (8\%), and drop (5\%) compared to EE-HRP (22) and MAREERP (23).

The rest of this paper is organized as follows: Section 2, reports on Literature survey. Section 3, presents a review on "K-Means-AODV-ACO" Methodology in MANET. Section 4, describes the results and discussion of overall "K-Means-AODVACO" methodology.

\section{Literature review}

G. Singh, N. Kumar, and A. K. Verma [17] introduced the Ant Colony Optimization (ACO) based Routing Algorithm (ANTALG) that considered the random election of the transmitter (source) and Receiver (Destination) nodes and also it replaced the agents among them. The nodes trip time was recorded by creating the pheromone tables and data structures while moving the ants (agents). ANTLAG algorithm had the best pherome value in their agents and this algorithm used the global updation to identify the effective route. The network declares the transmission path as a broken path, when the transmission time of HELLO packet is more. The security features of communication among the nodes not performed.

S. Chettibi, and S. Chikhi [18] presented a RP called as Dynamic Fuzzy Energy State based AODV (DFES-AODV). The forwarding probability of route requests was decided by using the Mamdani fuzzylogic system in each node. The mobile node's residual battery level and the energy drain rate were the inputs for the FLS. In addition, an FSARSAAODV algorithm was implemented with the fuzzy extension of the critic architecture for SARSA RL algorithm. This DFES-AODV is an energy efficient method and it is self-adaptive. FSARSA-AODV requires an empirical adjustment of several functional parameters. Number of nodes/user are less in the simulation.

W. Liu, and W. Kim [19] presented the Stability Considered Density Adaptive Routing (SDR) in MANET to create a stable route and it reduced the routing overhead. In SDR various routing tactics were performed according to the related density of vicinity and this routing was selected as two modes. Those are dense mode (D-mode) and sparse mode (S-mode). The routing overhead minimized by considering the stability value of each node while performing re-routing.

R. M. Kuchaki, and H. Fatemidokht [20] adopted the nature inspired (swarm intelligence) algorithms named as FBeeAdHoc for offering the secure routing among the MANET. The attacks from the malicious nodes were overcome by FbeeAdHoc secure routing protocol. Transmission efficiency of FbeeAdHoc is less compared to the BeeAdHoc algorithm. The optimizing of fuzzy membership function and finding selfish node is to be further enhanced.

W. Jabbar, A. M. Ismail, and R. Nordin [21] presented the Multi-Path Battery and Mobility Aware Routing scheme (MBMA-OLSR) which is influenced by the MP-OLSR. The metric of Multi 
Criteria Node Rank (MCNR) was used in the MBMA-OLSR and this MCNR includes the node's speed and the residual battery energy. The stability of the links was ranked by link assessment function and then it selected the stable and efficient path for data transmission. MBMA-OLSR used an Energy and Mobility Aware Multi Point Relay (EMA-MPR) selection mechanism for locating the nodes inclination. The energy efficiency was achieved by transmitting more packets at low energy cost per packet. The work doesn't evaluate in the large-scale network deployments and also in similar multi-hop networks such as WSNs and WMNs.

\section{Kmeans-AODVACO methodology}

The nodes in the MANET have limited power capabilities; it leads to link failure while transmitting the data packets from source to destination. In order to overcome that, the k-means clustering with AODV routing is utilized in the MANET. Then the AODV routing is optimized with the help of ACO to select the optimal path to send the data packets via the network. The overall process of this proposed scheme is given in the flowchart as shown in Fig. 1.

- Initially, the sensor nodes are arbitrarily organized in the interested area.

- K-means clustering is applied in the sensor nodes to cluster the network into a number of clusters. Then the Cluster-head is selected from each cluster.

- AODV with ACO routing is applied to create the optimal path from source to destination.

- From the routing, an optimized node is selected to form the route from source to destination.

- If the selected node has required energy to transfer the data means, it delivers the data from one node to another node or else it selects another optimal node for transferring the information.

- After creating the path from source-todestination, it sends the data to the destination.

- This proposed method predicted the optimal path for data transfer under the power constraint nodes in a MANET.

- At last, the performance of this proposed method is analysed in terms of throughput, end-to-end delay, and packet-delivery-ratio and energy consumption.

The following session summarizes the "Kmeans-AODVACO" methodology.

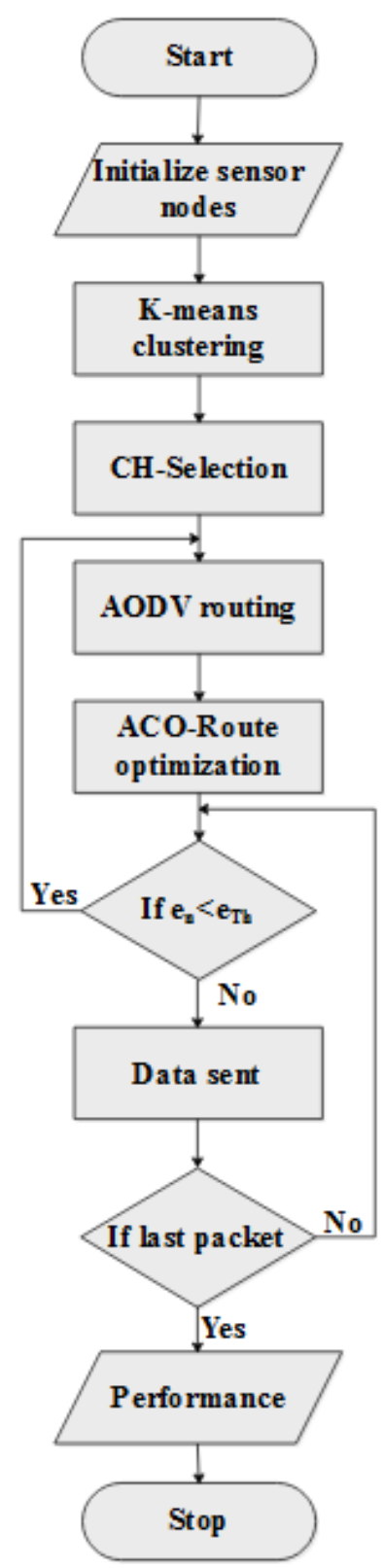

Figure. 1 Flowchart for the proposed scheme

\subsection{Kmeans clustering}

K-Means might be computationally quicker than hierarchical grouping with larger number of variables by producing tighter clusters than hierarchical clustering. K-means algorithm is followed three steps.

Initialisation: Set common centre points

- Assign each object to the cluster with the closest centre point

- Calculate centre points as the centroids of the clusters of the current divider (i.e. mean point of the cluster)

- Turn back to step 1, stop when no newer obligation of nodes.

The Fig. 2 describes about the K-means clustering algorithm. 


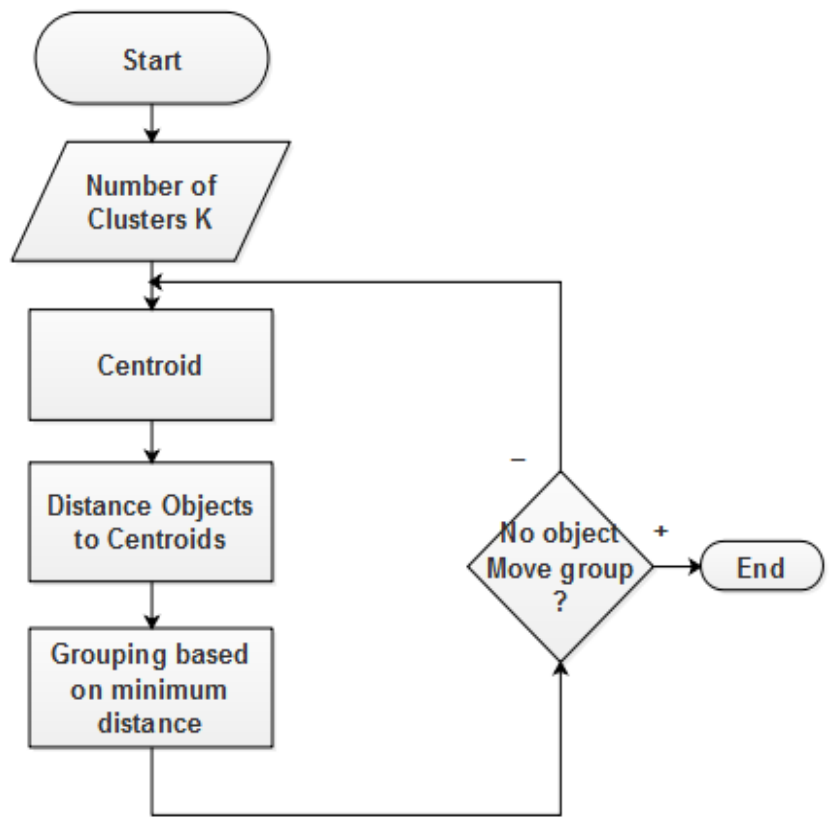

Figure. 2 K-Means algorithm

The clustering algorithm makes the transmission in a local domain and transmit the forwarding nodes (gateway-nodes). A group of nodes forms a cluster and the local co-operations between cluster members that are controlled through a $\mathrm{CH}$. The following steps designates, how the clustering of networks takes place;

A $k$ number of clusters are produced from the $n$ number of SNs, the fitness function is minimized by the algorithm. The fitness function that is used in this k-means clustering is squared error function and it is given in Eq. (1).

$$
F=\sum_{j=1}^{k} \sum_{i=1}^{n}\left\|x_{i}-c_{j}\right\|^{2}
$$

Where, the centre of $j^{\text {th }}$ cluster is represented as $c_{j}$, data point of $i^{t h}$ sample is denoted as $x_{i}$ and the distance from the each $\mathrm{SN}$ to the cluster centre is represented by $\left\|x_{i}-c_{j}\right\|^{2}$.

There are four main steps performed in K-means clustering algorithm.

\section{Step 1:}

Firstly, the $k$ clusters are formed from the SNs by compelling the $k$ number of centroids at random places.

\section{Step 2:}

The Euclidean distance from each $\mathrm{SN}$ to the centroid is computed for making the $k$ initial clusters. Consider each node is closest to the centroid. The Euclidean distance from one node to another node is given in Eq. (2). $\frac{\text { Eucildean distance }=}{\sqrt{\left(x_{1}-x_{2}\right)^{2}+\left(y_{1}-y_{2}\right)^{2}}}$

Where, the co-ordinates of $x$ and $y$ axis is represented as $x_{1}, x_{2}$ and $y_{1}, y_{2}$ respectively.

\section{Step 3:}

The situation of every node is checked from the previous position and each cluster areas are again generated in a network.

\section{Step 4:}

If the location of centroid becomes changed, then again go to step 2 for attaining the efficient clusters else the clustering progression to end. At last, the centroid, which is assigned from the $\mathrm{K}$ means clustering as an ideal $\mathrm{CH}$ for a group in the networks.

After the clustering of nodes, the routing process takes place using AODV Routing Protocol, which is explained below.

\subsection{AODV routing protocol}

The AODV is a reactive routing protocol; subsequently routes are resolved only when needed. Fig. 3 exhibits the message exchanges of the AODV RP. Hello messages might be employed by every active node in which its occasionally broadcasts a Hello message to its neighbouring nodes. Since nodes occasionally send Hello messages, if a node neglects to get a few Hello messages from a neighbour, a connection break is distinguished. Exactly when a source has data to transmit messages to an unidentified destination, it conveys a RREQ for that destination. A RREQ message receive a route from the source that is created at each intermediate node. If the receiving node has not received this RREQ previously, then it is not the destination and does not have a current course to the destination that it rebroadcasts the RREQ. In the event that the accepting node is the destination or has a current course to the destination, it delivers a RREP. The RREP is uni-cast in a hop-by-hop form to the source. Each intermediate node creates a route to the destination as the RREP stimulates. It records the route to the destination and can start sending information, when the source gets the RREP. It generates a RREP, if the receiving node is the destination node or has a current route to the destination. Each intermediate node makes a route to the destination as the RREP engenders. The route with the shortest path is chosen if different RREPs are received from the source. 


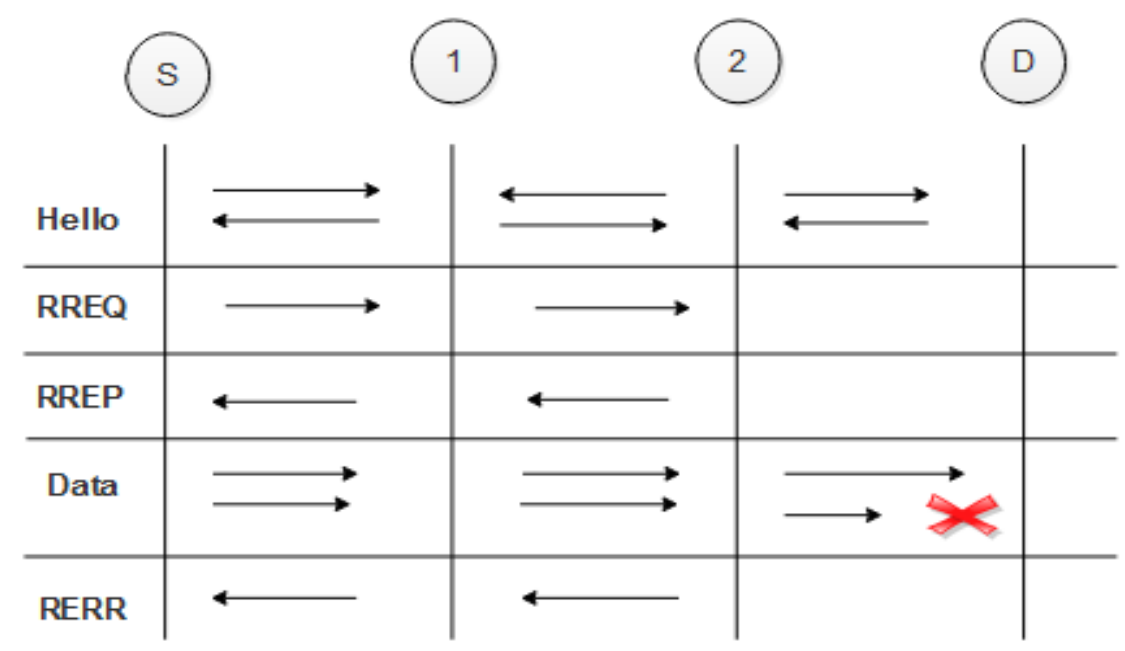

Figure. 3 AODV protocol message

Each node along the route informs the timers linked with the routes to the source and destination by maintaining the routes in the routing table. A node cannot make certain route updates, whether the route is still valid accordingly by expelling the route from its routing table. A RERR is sent to the source of the information in a hop-by-hop fashion, if the information is streaming and a connection break is recognized. Each intermediate node invalidates route to any unreachable destinations as the RERR broadcasts towards the source. When the source of the data/information admits the RERR, it reversals the route and reinitiates route discovery if essential. AODV routing protocol is optimized using ACO.

\subsection{Route optimization using ACO}

ACO is a part of the larger field of swarm intelligence in which scientists study the behaviour patterns of bees and other social insects in order to simulate processes. The main source of motivation is found in the capacity of certain kinds of ants to search the shortest path between their nest and a food sources using Pheromone (Impulsive Chemical Substance). Ant leave traces of pheromone as they migrate between sources to destination. Ants specially go in the course of high pheromone intensities in search of food. The higher levels of pheromone is received, when minimum paths are finished faster. The positive strengthening process allows the colony as a whole to touch on the shortest path.

\section{Results and discussions}

The "K-Means-AODV" method is implemented in the NS2 simulation tool. The simulations parameters are shown in Table 1. The simulation starts and end time is denoted as 0.0001-50.0000 correspondingly by varying the number of static nodes as 20, 40, 60, 80 and 100. The MAC Type is 802_11 with Omni Antenna model. This section gives a detailed view of the results that are obtained using "K-Means-AODV" method for providing efficient energy and ACO optimization, which reduces the total energy consumption in the network by minimizing the lifetime in mobile ad-hoc networks by giving better performance compared with the existing methods. The performance is calculated by measuring the delay, routing overhead, energy and drop parameters. The performance metrics is given below.

\subsection{Packet delivery ratio (PDR)}

Based on a total amount of packets established in a ratio by a total number of destination packet sent by the source node, which is given in Eq. (3).

$$
\text { DelRatio }=\frac{\text { (no. of packets send }- \text { packets lost) }}{\text { no. of packets send }}
$$

\subsection{Energy consumption}

A number of nodes is equivalent to the amount of received energy consumption. A node drops a specific amount of energy for every packet transmission and received, which is given in Eq. (4).

$$
\text { Energy }=\frac{\text { amount of energy for every packets }}{\text { total simulation time }}
$$

\subsection{Delay}

The difference between sending time of packets and receiving time of packets is known as delay, which is given in Eq. (5). 
Delay $=$ Time spend on Hop $1+$ time spend on Hop $2+$ ..... +time spend on Hop $n$

\subsection{Packet drop/packet loss}

Total amount of packets sends and packet established is known as the packet drop/packet loss, which is given in Eq. (6).

$$
\text { Drop = }
$$

(Total no.of packets send-Packet received) Total number of simulation

Table 1 shows the delay by changing different nodes such as 20, 40, 60, 80, and 100 of fixed Nodes. Hence, K-Means-AODV" Methodology shows better results than that EE-HRP and MAREERP.
The evaluation of nodes vs. delay between Kmeans-AODV-ACO and existing method is plotted in Fig. 4. The value of delay is diminished in Kmeans-AODV-ACO method compared to the EEHRP method with different 20, 40, 6080 and 100 Nodes.

Table 2 shows the DelRatio by changing different nodes such as 20, 40,60, 80, and 100 of fixed Nodes. Hence, K-Means-AODV-ACO" Methodology shows better results than that EE-HRP and MAREERP.

The Evaluation of Nodes vs. DelRatio between Kmeans-AODV-ACO and existing method is plotted in Fig. 5. The value of DelRatio is increased in Kmeans-AODV-ACO method compared to the EE-HRP method with different 20, 40, 6080 and 100 Nodes.

Table 1 . Delay by varying number of nodes

\begin{tabular}{|c|c|c|c|c|c|}
\hline QoS & \multicolumn{5}{|c|}{ Delay } \\
\hline Nodes & $\mathbf{2 0}$ & $\mathbf{4 0}$ & $\mathbf{6 0}$ & $\mathbf{8 0}$ & $\mathbf{1 0 0}$ \\
\hline EE-HRP [22] & 1.656479 & 3.106972 & 3.532987 & 4.324796 & 4.523796 \\
\hline MAREERP [23] & 1.312789 & 2.245609 & 2.689076 & 3.107033 & 3.888776 \\
\hline Kmeans -AODV & 1.179187 & 1.359974 & 1.801087 & 1.184184 & 1.683421 \\
\hline
\end{tabular}

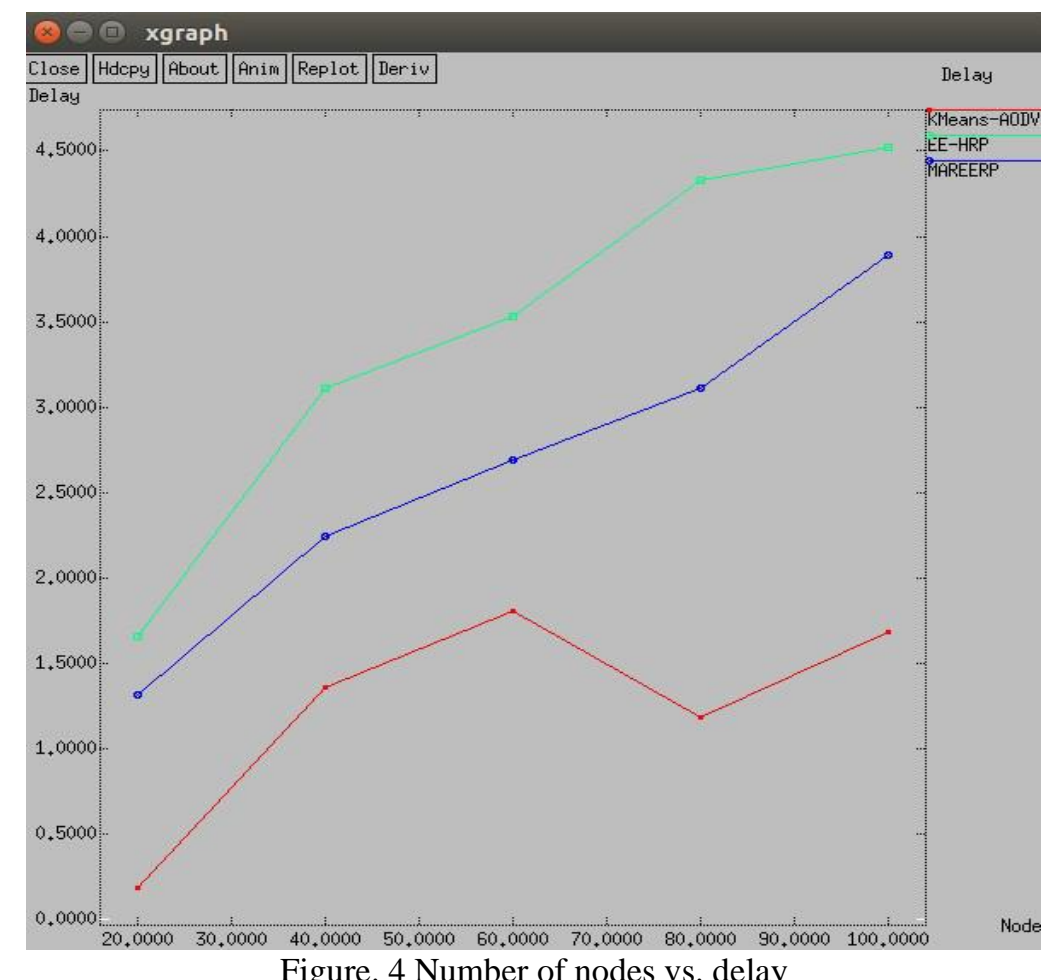

Table 2. DelRatio by varying number of nodes

\begin{tabular}{|c|c|c|c|c|c|}
\hline QoS & \multicolumn{5}{|c|}{ DelRatio } \\
\hline Nodes & $\mathbf{2 0}$ & $\mathbf{4 0}$ & $\mathbf{6 0}$ & $\mathbf{8 0}$ & $\mathbf{1 0 0}$ \\
\hline EE-HRP [22] & 332.73 & 259.05 & 229.35 & 210.37 & 112.67 \\
\hline MAREERP [23] & 334.56 & 267.89 & 245.78 & 229.89 & 168.78 \\
\hline Kmeans -AODV & 382.75 & 289.95 & 265.35 & 237.38 & 197.75 \\
\hline
\end{tabular}


Table 3 shows the Energy by changing different nodes such as 20,40,60, 80, and 100 of fixed Nodes. Hence, K-Means-AODV" Methodology shows better results than that EE-HRP and MAREERP.

The Evaluation of Nodes vs. energy between Kmeans-AODV-ACO and existing method is plotted in Fig. 6. The value of energy is decreased in Kmeans-AODV-ACO method compared to the EEHRP method with different 20, 40, 6080 and 100 Nodes.

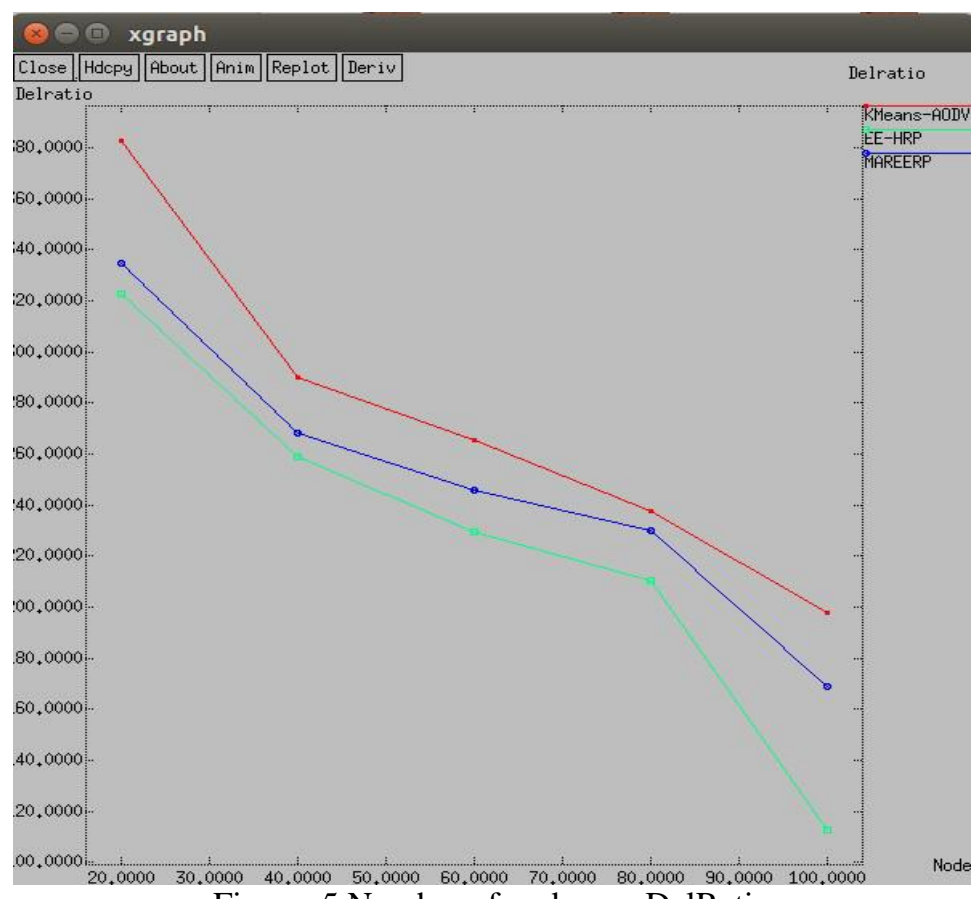

Figure. 5 Number of nodes vs. DelRatio

Table 3. Energy Consumption by varying number of nodes

\begin{tabular}{|c|c|c|c|c|c|}
\hline QoS & \multicolumn{5}{|c|}{ Energy Consumption } \\
\hline Nodes & $\mathbf{2 0}$ & $\mathbf{4 0}$ & $\mathbf{6 0}$ & $\mathbf{8 0}$ & $\mathbf{1 0 0}$ \\
\hline EE-HRP [22] & 24.241127 & 22.431217 & 22.217316 & 22.176739 & 22.567896 \\
\hline MAREERP [23] & 24.17114 & 22.16345 & 21.94356 & 22.06782 & 22.34789 \\
\hline Kmeans -AODV & 24.062211 & 22.011249 & 21.725402 & 21.974539 & 22.238232 \\
\hline
\end{tabular}

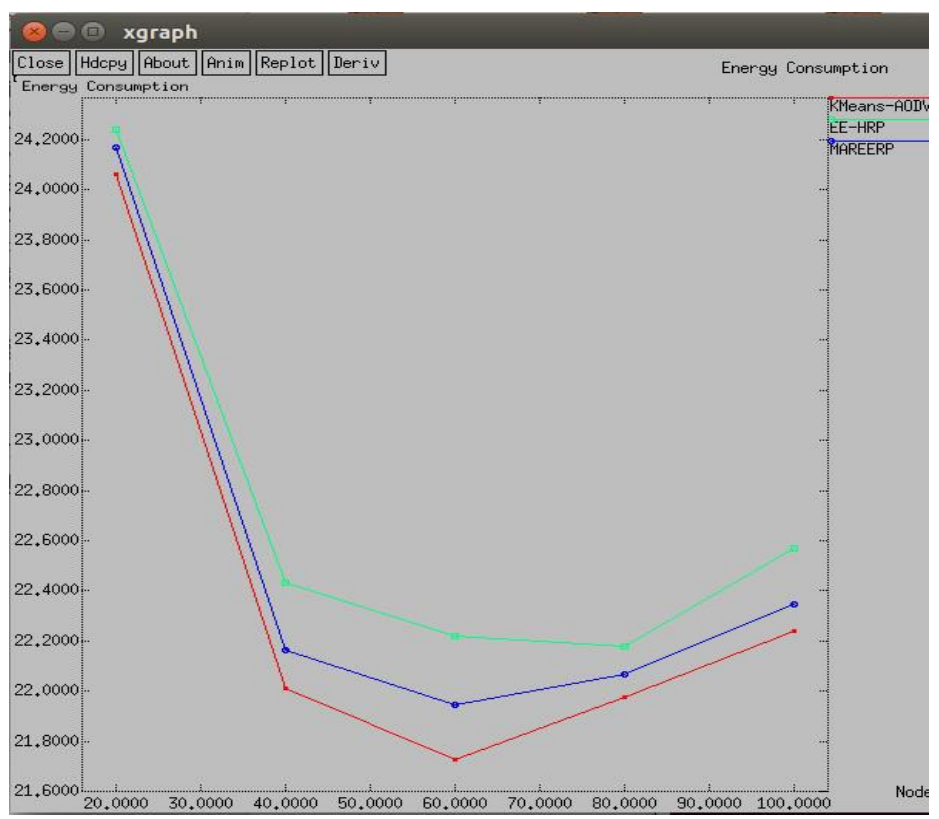

Figure. 6 Number of nodes vs. energy 
Table 4. Drop by varying number of nodes

\begin{tabular}{|c|c|c|c|c|c|}
\hline QoS & \multicolumn{5}{|c|}{ Drop } \\
\hline Nodes & $\mathbf{2 0}$ & $\mathbf{4 0}$ & $\mathbf{6 0}$ & $\mathbf{8 0}$ & $\mathbf{1 0 0}$ \\
\hline EE-HRP [22] & 1267 & 1226 & 1251 & 1345 & 1366 \\
\hline MAREERP [23] & 1290 & 1256 & 1278 & 1373 & 1386 \\
\hline Kmeans -AODV & 1256 & 1204 & 1235 & 1302 & 1350 \\
\hline
\end{tabular}

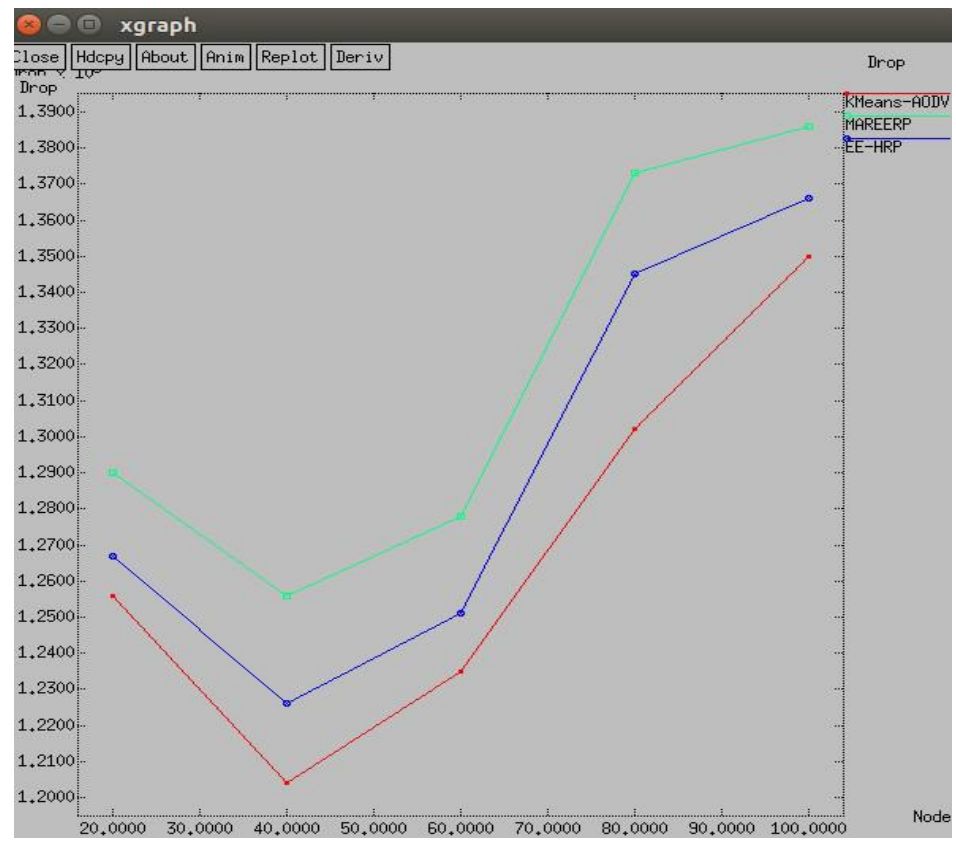

Figure. 7 Number of nodes vs. drop

Table 5. Simulation Parameters of K-Means-AODVACO

\begin{tabular}{|c|c|}
\hline Clustering technique & K-Means \\
\hline Routing technique & AODV \\
\hline Optimization techniques & ACO \\
\hline Simulator used & NS2 \\
\hline Simulation start time & 0.0000000001 \\
\hline Simulation End time & 50.000000000 \\
\hline Number of mobile nodes & $20,40,60,80$ and 100 \\
\hline Antenna Model & Omni Antenna \\
\hline Minimum speed & 28 ms \\
\hline Network Interface types & Wireless \\
\hline MAC Type & MAC/802_11 \\
\hline Initial Transmit Power & 0.660 \\
\hline Initial Receive Power & 0.395 \\
\hline
\end{tabular}

Table 4 shows the drop by changing different nodes such as 20, 40, 60, 80, and 100 of fixed Nodes. Hence, K-Means-AODV" Methodology shows better results than EE-HRP and MAREERP.

The Evaluation of Nodes vs. drop between Kmeans-AODV-ACO and existing method is plotted in Fig. 7. The value of delay is decreased in Kmeans-AODV-ACO method compared to the EEHRP method with different 20, 40, 6080 and 100 Nodes.

The simulation parameters are shown in Table 5. The simulation starts and end time are denoted as 0.0001-50.0000 respectively by varying the number of static nodes as 20,40, 60, 80 and 100 . The MAC Type is 802_11 with Omni Antenna model

\section{Conclusion}

This section describes the conclusion of the proposed energy conservation mechanism. The energy is calculated based on the prediction of the energy consumption level of the node. The energy computation method is performed using the Kmeans clustering along with the AODV routing with ACO optimization by selecting the shortest path in the network. The "Kmeans-AODV-ACO" model handles network state related constraints such as energy consumption of nodes in the ad-hoc networks. The performance of the proposed " $\mathrm{K}$ Means-AODV-ACO" model is compared with the existing EE-HRP models and MAREERP. The proposed model achieved better PDR (7\%), end-toend delay $(6 \%)$, drop (5\%) and energy consumption $(8 \%)$ in ad-hoc networks. Improvement in the choosing shortest path in ad-hoc networks can be established in the future improvement.

\section{References}

[1] S.A. Ade and P.A. Tijare, "Performance comparison of AODV, DSDV, OLSR and DSR 
routing protocols in mobile ad hoc networks", International journal of information technology and knowledge management, Vol.2, No.2, pp.545-548, 2010.

[2] P.F.A. Selvi and M.S.K. Manikandan, "Ant based multipath backbone routing for load balancing in MANET", IET Communications, Vol.11, No.1, pp.136-141. 2017

[3] R.C. Biradar and S.S. Manvi, "Neighbor supported reliable multipath multicast routing in MANETs", Journal of Network and Computer Applications, Vol.35, No.3, pp.1074-1085, 2012.

[4] S. Gopinath and N. Nagarajan, "Energy based reliable multicast routing protocol for packet forwarding in MANET", Journal of applied research and technology, Ant based multipath backbone routing for load balancing in MANET, Vol.13, No.3, pp.374-381, 2015.

[5] J. Sathiamoorthy and B. Ramakrishnan, "Design of a proficient hybrid protocol for efficient route discovery and secure data transmission in CEAACK MANETs", Journal of Information Security and Applications, Vol.36, pp.43-58, 2017.

[6] R.M. Nareshkumar, S. Phanikumar, and M.K. Singh, "Intelligent Routing in MANET Using Self-Adaptive Genetic Algorithm", In: Proc. of International Conf. On Advances in Systems, Control and Automation, Springer, Singapore, pp.595-603, 2018.

[7] P. Zhao, X. Yang, W. Yu, and X. Fu, "A loosevirtual-clustering-based routing for power heterogeneous MANETs", IEEE Transactions on vehicular technology, Vol.62, No.5, pp.2290-2302, 2013.

[8] W. Liu and M. Yu, "AASR: authenticated anonymous secure routing for MANETs in adversarial environments", IEEE transactions on vehicular technology, Vol.63, No.9, pp.4585-4593, 2014.

[9] H. Le Minh, G. Sexton, and N. Aslam, "Selfadaptive proactive routing scheme for mobile ad-hoc networks", IET Networks, Vol.4, No.2, pp.128-136, 2014.

[10] K. Sumathi and A. Priyadharshini, "Energy optimization in magnets using on-demand routing protocol", Procedia Computer Science, Vol.47, 460-470, 2015.

[11] S.Y. Han and D. Lee, "An adaptive hello messaging scheme for neighbor discovery in on-demand MANET routing protocols", IEEE communications letters, Vol.17, No.5, pp.10401043, 2013.

[12] S.K. Das and S. Tripathi, "Energy efficient routing protocol for manet based on vague set measurement technique", Procedia Computer Science, Vol.58, pp.348-355, 2015.

[13] S. Jamali, L. Rezaei, and S.J. Gudakahriz, "An energy-efficient routing protocol for MANETs: a particle swarm optimization approach", Journal of applied research and technology, Vol.11, No.6, pp.803-812, 2013.

[14] K. Tamizarasu and M. Rajaram, "An AODVbased Clustering Approach for Efficient Routing in MANET", International Journal of Computer Applications, Vol.51, No.15, 2012.

[15] H. Shen and L. Zhao, "ALERT: an anonymous location-based efficient routing protocol in MANETs", IEEE transactions on mobile computing, Vol.12, No.6, pp.1079-1093, 2013.

[16] M. Uddin, A. Taha, R. Alsaqour, and T. Saba, "Energy Efficient Multipath Routing Protocol for Mobile ad-hoc Network Using the Fitness Function", IEEE access, Vol.5, pp.1036910381.

[17] G. Singh, N. Kumar, and A.K. Verma, "Antalg: An innovative aco based routing algorithm for manets", Journal of Network and Computer Applications, Vol.45, pp.151-167, 2014.

[18] S. Chettibi and S. Chikhi, "Dynamic fuzzy logic and reinforcement learning for adaptive energy efficient routing in mobile ad-hoc networks", Applied Soft Computing, Vol.38, pp.321-328, 2016.

[19] W. Liu and W. Kim, "A stability-considered density-adaptive routing protocol in MANETs", Journal of Systems Architecture, Vol.59, No.9, pp.767-775, 2013.

[20] R.M. Kuchaki and H. Fatemidokht, "FBeeAdHoc: A secure routing protocol for BeeAdHoc based on fuzzy logic in MANETs", AEU-International Journal of Electronics and Communications, Vol.69, No.11, pp.1613-1621, 2015.

[21] W. Jabbar, A.M. Ismail, and R. Nordin, "Energy and mobility conscious multipath routing scheme for route stability and load balancing in MANETs", Simulation Modelling Practice and Theory, Vol.77, pp.245-271, 2017.

[22] S. Ghode and K. Bhoyar, "Optimized Energy Efficient Hybrid Routing Protocol (EE-HRP) for Minimum Battery Power Consumption in MANET", International Journal of Intelligent Engineering and Systems, Vol.11, No.5, pp.5973,2018

[23] S. Nallusamy, S. Appavupillai, and S. Ponnusamy, "Mobile Agents based Reliable and Energy Efficient Routing Protocol for MANET", International Journal of Intelligent 
Engineering and Systems, Vol.9, No.3, pp.110-

116, 2016 